\title{
The impact of work-related stress on the cognition domain of executive functioning of health care workers during the COVID-19 pandemic
}

\author{
Sahar Ali Farahat ${ }^{1} \cdot$ Omnia Raafat Amin ${ }^{2} \cdot$ Hatem Sherif Hamdy ${ }^{1} \cdot$ Marwa Mohammed Fouad $^{1}$ (D)
}

Received: 7 August 2021 / Accepted: 25 October 2021 / Published online: 3 November 2021

(c) The Author(s), under exclusive licence to Springer-Verlag GmbH Germany, part of Springer Nature 2021

\begin{abstract}
Purpose The current study addressed work-related stress and burnout experienced by health care workers (HCWs) in Cairo University isolation hospital and its impact on the cognitive domain of their executive functioning.

Methods Arabic validated questionnaires of Beverly Potter for sources of work-related burnout and Maslach Burnout Inventory Human Services Survey for evaluation of burnout degree of $81 \mathrm{HCWs}$ were used. Wisconsin Card Sorting Test was used to examine the HCWs cognitive executive functioning during hospital work and 2 weeks after home isolation.

Results A high degree of work-related stress was evident by a high score on Beverly questionnaire with a mean \pm SD of $(132.08 \pm 12.573)$. A high degree of burnout was marked with a mean \pm SD of (28.48 \pm 6.622$)$ for Emotional Exhaustion module, (31.85 \pm 5.439$)$ Personal Achievement, and (17.52 \pm 6.707$)$ Depersonalization. Health care workers experienced impairment of executive functioning in the form of increased total errors of Wisconsin Card Sorting Test during hospital shifts compared to 2 weeks after. There is a positive significant correlation between work-related stress level and Maslach Burnout Inventory and the total errors of Wisconsin Card Sorting Test results during the hospital stay $(p=<0.001)$, as well as 2 weeks after $(p=<0.001)$. This cognitive decline manifested in increased errors during HCWs' clinical work.

Conclusions Health care workers on the front line experienced a high degree of work-related stress in addition to burnout in the form of Emotional Exhaustion, Depersonalization, and reduced Personal Achievement. They also suffered from impaired cognitive executive functioning due to such stressful exposure.
\end{abstract}

Keywords COVID-19 · Health care workers · Isolation hospital · Work-related stress · Burnout · Cognitive domain · Executive functioning

Marwa Mohammed Fouad

marwa.fouad@kasralainy.edu.eg

Sahar Ali Farahat

sahar.farahat@kasralainy.edu.eg

Omnia Raafat Amin

omniaraffat@yahoo.com

Hatem Sherif Hamdy

hatem.s.hamdy@gmail.com

1 Department of Occupational \& Environmental Medicine, Kasr Al-Aini Faculty of Medicine, Cairo University, Cairo, Egypt

2 Department of Psychiatry, Kasr Al-Aini Faculty of Medicine, Cairo University, Cairo, Egypt

\section{Introduction}

Since late December 2019, the outbreak of a new Corona Virus Disease (COVID-19), previously known as the novel Corona Virus (nCoV-2019) was reported in Wuhan, China. In April 2020, the disease had spread into more than 60 countries around the world, which marked the beginning of the COVID-19 pandemic with more than one million cases of infected patients (Wu and McGoogan 2020).

Since then, the workload raised drastically on health care workers (HCWs), requiring them to work in isolation hospitals for COVID-19 positive patients. Different periods of hospital stay ranging from 1 week to 1 month were arranged for HCWs within the COVID-19 isolation hospitals to face the increasing influx of infected patients. This long hospital stay made HCWs susceptible to high level of work-related stress, specifically 
with having greater risk of being exposed to infection (Elshaer et al. 2018; Liu et al. 2020; Ng et al. 2020).

Physicians and nurses working in the COVID-19 isolation hospital face a unique sort of work-related stress that includes new risk factors that should be investigated, such as dealing with critically sick patients who have an often unpredictable disease course, high mortality rates and lack of effective treatment or definite treatment guidelines, stigmatization, feeling rejected from neighborhood and fear of transmitting the infection to their families (Carmassi et al. 2020).

The symptoms of work-related stress can encompass a wide spectrum of psychiatric illnesses as sleep disorders and anxiety, as well as medical complaints as neck and lower back pain. Chronic work-related stress can especially lead to conditions like depression, burnout, and exhaustion disorders (Maslach et al. 2001; Bonde 2008; Grynderup et al. 2013; Hasselberg et al. 2014; Yu et al. 2015).

Some studies have pointed out the possibility of occurrence of cognitive impairment among workers experiencing work stress and burnout (Österberg et al. 2009; Jonsdottir et al. 2013; Deligkaris et al. 2014). Individuals suffering from work-related stress also complain of cognitive impairments, affecting their memory and concentration namely, prospective memory, processing speed, and complex working memory. However, it remains uncertain if these complaints reflect an actual decline in cognitive functions that can be measured by neuropsychological testing (Deligkaris et al. 2014).

Several previous studies have used neuropsychological testing in an attempt to discover whether workers with workrelated stress have cognitive impairments that are objectively measurable. However, the results are relatively inconsistent (Österberg et al. 2009; Jonsdottir et al. 2013). Cognitive impairment among HCWs may be reflected on their work with subsequent errors and increased risk of infection transmission (Salam et al. 2019).

Most of the recent research focused on the assessment of the degree of work-related stress, anxiety, and depression among health care workers during COVID19 pandemic, while the effect of work-related stress on cognitive function have not been widely studied, therefore, this study aimed to assess the degree of work-related stress and its sources in addition to measuring different areas of burnout and their impact on the cognitive impairment among HCWs in isolation hospitals where the highest level of perceived stress might be there.

\section{Methods}

\section{Study design and data collection}

The current study is a prospective cohort study assessing the work-related stress, burnout and executive functioning of
81 health care workers during their work in the COVID-19 Cairo University isolation hospital from May 2020 to August 2020. Since there are no available data regarding assessment of the degree of impairment of cognitive function, specifically executive function, as a result of exposure to workrelated stress during the period of COVID-19 Pandemic, pilot study was conducted to assess the degree of cognitive function affection in physician compared to nurses. Based on the results of the pilot study, comparing physician to nurses with ratio $1: 1$, the average WCST in physician was 2.37 with standard deviation 1.918 while the average WCST in nurses was 8.4 with standard deviation 3.5 , so we will need to study 13 participants per group, it will be compensated by $15 \%$ due to the use of non-parametric tests, and compensated by $30 \%$ for suspected losses so the final sample size will be 20 subjects per group (total 40 participants) to be able to reject the null hypothesis that the population means WCST in physicians and nurses are equal with probability (power) 0.99 . The Type I error probability associated with the test of this null hypothesis is 0.01 . Sample size was calculated using $G$ power program.

Out of approximately $140 \mathrm{HCWs}, 81$ (50 physicians and 31 nurses) have agreed to be interviewed and assessed by below-mentioned methods during their break. This study has been conducted in one of isolation hospital affiliated to Cairo University hospitals over three rotation schedules, each is 2 weeks long and separated by 1 week, during the period from (April 2020-June 2020) i.e., during the 1st wave of COVID-19 in Egypt. It was conducted on two occupational groups of health care workers, namely physicians and nurses. Physicians spend 1 week in the isolation hospital without leaving, while nurses spend 2 weeks. Health care workers in the isolation hospital with age range of 25-52 years were included in the study. Any health care worker with current neurological disorder, current psychiatric disorders, color blindness, advanced chronic diseases or current use of any psychotropic drugs is excluded from the study.

The study was conducted through face-to-face structured interviews during their breaks in their rooms using the questionnaires listed below after explaining the different components. Afterward every participant was assessed using Wisconsin Card Sorting Test. After the end of their hospital stay, they were advised to isolate themselves for 2 weeks. After these 2 weeks of home isolation, a re-assessment of the executive functioning was done to observe any difference in cognitive performance. All participants were subjected to full history taking including personal, present, past, family, and occupational history with special emphasis on detailed neurological and psychiatric history. Full examination was performed with special focus on neurological examination. All the study participants were offered a written consent to take part in the study after explaining the importance of this study. The Research Ethics Committee, Faculty of 
Medicine, Cairo University has approved the study with Code MS-373-2020.

\section{Work-related stress assessment}

The Arabic version of Beverly Potter questionnaire (Potter 2009) translated and validated by Abdelkader (2010) of sources of work-related burnout was used to assess the degree of work-related stress of HCWs in the COVID-19 isolation hospital. It was chosen as it is the only one that has validated Arabic version with Egyptian slang prepared by Farag Abdelkader. It is a 48-item in Arabic language measuring sources and degree of work stress. It encompasses 12 modules of questions tackling different sources of stress in the workplace as follows: workload, decision latitude, conflicting tasks, feedback, managerial and organizational transparency, team work and co-workers relationships, reward, punishment, moral dilemmas, accessibility of information, alienation, and boredom.

Every module contains four questions. Every question can either be answered in its frequency of occurrence with possible scores from 4 ('always), 3 ('often'), 2 ('rare'), 1 ('never). The sum of the score of all modules would have the least result of 48, when the participant would answer only 'never' for every question, i.e., $48 \times 1$, while the maximum score would be 192 if only 'always' would be the answer, i.e., $48 \times 4$.

Any participant score that is above $97(t$ score $=50)$ is suffering from work-related stress. The higher the score, the higher the degree of stress is.

\section{Burnout assessment}

Occupational burnout in health care workers working in the COVID-19 isolation hospital was evaluated using the Arabic version of Maslach Burnout Inventory-Human Services Survey for Medical Personnel (MBI-HSS) (Abd-Allah and El-Hawy 2019). It comprises 22 items regrouped into three subscales: Emotional Exhaustion (EE; nine items), Depersonalization (DP; five items), and Personal Accomplishment (PA; eight items). Each item can be answered on a sevenpoint scale ranging from "never" $(=0)$ to "daily" $(=6)$. The results of this inventory consist of three separate scores, one for each factor. A combination of high scores on EE and DP, and a low score on PA, correspond to a high level of burnout.

Emotional Exhaustion (EE) addresses the fatigue, emotional drain, frustration, and loss of hope in the face of increased workload. Low-level burnout $\leq 17$, moderate burnout 18-29, high-level burnout $\geq 30$.

Depersonalization (DP) confronts loss of empathy experienced by HCWs and the feeling of their detachment from the purpose of their work. Low-level burnout $\leq 5$, moderate burnout 6-11, high-level burnout $\geq 12$.
Personal Achievement Scale (PA) includes individual assessment regarding their work achievement. A reduction of Personal Achievement is a sign of burnout. Individuals assessing themselves negatively in this scale reflect the demotivating spirit of the HCWs. This predicts impending burnout. A total score of 33 or less indicates high-level burnout, between 34 and 39 inclusive is moderate burnout and greater than 40 is low-level burnout.

\section{Cognitive executive functioning}

Wisconsin Card Sorting Test (The 64 card version), a neuropsychological test that was used to measure the executive functioning of health care workers (Heaton and Staff 1993). The test consists of 64 different cards that can be distinguished by number, color or shape of the figures. Four stimulus cards were presented in front of the subject, for example the first with one red circle, the second with two green stars, the third with three blue crosses, and the fourth with four yellow triangles on it. One response, which has similar designs as stimulus cards but varying in color, shape and number is presented card one at a time in front of the subject. The subject was then told to match the response card to one of the four stimulus cards and was given feedback each time whether he or she was right or wrong. Every ten correct trials, the category would change. So, if the subject had to sort according to color, he/she would be asked to sort according to shape. There is no specific order of categories. The procedure continues until the subject had completed six sorting categories or until 64 response cards had been displayed.

Items that can be analyzed from this test includes: Total errors: the total number of incorrect responses, Number of categories completed: the number of sequences of ten consecutive correct matches, Perseverative errors: the number of items in which the participant persists in responding to incorrect stimulus characteristic and the Non-perseverative errors: the number of incorrect responses which are not due to a persistent response to a stimulus characteristic that is incorrect.

Due to the difficulty of conducting neuropsychological tests in a COVID-19 hospital since the presence of strict social distancing, wearing of PPE and stressed circumstances, only one reliable item of WCST has been chosen, namely total errors, for its easy calculation and administration.

There are no specific normative data available for Egypt or the Middle East to compare to. Normative data differ from individuals according to studies done all over the world. A study was done in India by Kohli and Kaur (2006) has also concluded that normative data are different in the Indian sample in comparison to the Western sample. 


\section{Statistical analysis}

The clinical data and measurements were statistically analyzed using Statistical computer software package SPSS v. 19.0. Mean and standard deviation were calculated for continuous variables while frequency and percent were calculated for categorical variables to compare continuous variables. Student $t$ test was used to estimate the probability of difference between parametric data. Wilcoxon signed-rank test and Mann-Whitney $U$ test were used for non-parametric statistical analysis for paired differences. Spearman's rank correlation coefficient was used for correlation studies. Statistical significance was considered when $p$ values are less than 0.05 .

\section{Results}

The studied population included $81 \mathrm{HCWs}, 52 \%(n=42)$ were males, $62 \%$ were physicians while $38 \%$ were nurses and $48 \%$ were married. The age of the study participants ranged from 25 to 52 , mean $\pm \mathrm{SD}(32.84 \pm 6.8)$.

The questionnaire on work-related stress among the study participants had a mean \pm SD of $(132.08 \pm 12.573)$, which indicates a high degree of work stress. Maslach Burnout Inventory for Human Health Services has shown a mean \pm SD of $(28.48 \pm 6.622)$ for the module of Emotional Exhaustion, (31.85 \pm 5.439$)$ for Personal Achievement, and $(17.52 \pm 6.707)$ for Depersonalization, which indicates a high degree of burnout. The total errors in the Wisconsin Card Sorting Test had a mean \pm SD of $(4.60 \pm 3.914)$ during the stay at the hospital and a mean \pm SD of $(2.21 \pm 2.728)$ 2 weeks after work with a significant statistical difference $(p<0.001)$ using Wilcoxon signed-rank test (Table 1).

Nurses had a statistically significant higher degree of work-related stress $(p<0.001)$ and a higher Emotional Exhaustion $(p<0.001)$ and Depersonalization $(p=0.014)$ in Maslach Burnout Inventory compared to physicians. They also had a statistically significant lower score in Personal Achievement Module of Maslach Burnout Inventory compared to physicians $(p=0.009)$. Physicians have shown a significant lower number of total errors in the WCST results compared to the nurses $(p<0.001)$. There is a significant difference between the experience of physicians and nurses $(p=0.009)$ (Table 2).

Married group of HCW had statistically non-significant higher degree of work-related stress compared to non-married group $(p=0.92)$. There is also a non-significant difference between the married and non-married group regarding the score of the Maslach Burnout Inventory. Married group also has shown a significant higher number of total errors in the WCST results compared to the non-married group $(p<0.001)$ (Table 3).

There was a non-significant difference in the degree of work-related stress, burnout and experience between male and female HCWs. However, there was a significant difference in total errors of WCST between them, in which females have a higher score of errors (Table 4).
Table 1 Mean \pm SD of questionnaire on work-related stress (WRS), Maslach Burnout Inventory Human Services Survey (MBI-HSS), and total errors of Wisconsin Card sorting Test in the hospital (WCST- during) and post-hospital stay (WCST-post) and the paired difference between the total errors of HCWs in WCST during their hospital stay (WCST-in) and 2 weeks after their work (WCST-post)

\begin{tabular}{|c|c|c|c|c|c|c|}
\hline & & & \multicolumn{4}{|c|}{ Participants $(N=81)$} \\
\hline & & & \multicolumn{3}{|l|}{ Mean } & $\pm \mathrm{SD}$ \\
\hline $\mathrm{WRS}^{\mathrm{a}}$ & & & \multicolumn{3}{|l|}{132.08} & 12.573 \\
\hline MBI-EE ${ }^{\mathrm{b}}$ & & & \multicolumn{3}{|l|}{28.48} & 6.622 \\
\hline MBI-PA $^{c}$ & & & \multicolumn{3}{|l|}{31.85} & 5.439 \\
\hline MBI-DP ${ }^{\mathrm{d}}$ & & & \multicolumn{3}{|l|}{17.52} & 6.707 \\
\hline & Mean & SD & $P$ value & Median & Range & \\
\hline Experience (years) & 10.52 & 9.435 & \multirow{3}{*}{$<0.001 *$} & 6 & $1-34$ & \\
\hline WCST (during) ${ }^{\mathrm{e}}$ & 4.60 & 3.914 & & 4 & $0-15$ & \\
\hline WCST (post) ${ }^{\mathrm{f}}$ & 2.21 & 2.728 & & 1 & $0-12$ & \\
\hline
\end{tabular}

${ }^{*} P$ value $<0.05$ is considered statistically significant

${ }^{\mathrm{a}}$ Work-related stress questionnaire

${ }^{b}$ Maslach Burnout Inventory for the module of Emotional Exhaustion

${ }^{c}$ Maslach Burnout Inventory for the module of Personal Achievement

${ }^{\mathrm{d}}$ Maslach Burnout Inventory for the module of Depersonalization

${ }^{\mathrm{e}}$ Wisconsin Card Sorting Test during hospital work

${ }^{\mathrm{f}}$ Wisconsin Card Sorting Test during after 2 weeks of home isolation 
Regarding the comparison between ICU/ER health care workers and ward admission (Zone) health care workers, there is a non-significant difference in the degree of workrelated stress, burnout and total errors in WCST between ICU/ER HCW and ward HCW (non-tabulated data).
Regarding the risk factors contributing to work-related stress, The highest-ranking risk factors were high workload (12\%) followed by lack of decision latitude $(10 \%)$, conflicting tasks (10\%), lack of feedback by their seniors
Table 2 Comparison of results of Mean \pm SD between physicians and nurses in results by work-related stress questionnaire (WRS), Maslach Burnout Inventory Human Services Survey (MBI-HSS), and total errors of Wisconsin Card Sorting Test in the hospital (WCST-during) and post-hospital stay (WCST-post)

\begin{tabular}{|c|c|c|c|c|c|c|c|c|c|}
\hline & \multicolumn{3}{|c|}{ Physicians $(N=50)$} & & \multicolumn{3}{|c|}{ Nurses $(N=31)$} & & \multirow[t]{2}{*}{$P$ value } \\
\hline & \multicolumn{2}{|c|}{ Mean } & \multicolumn{2}{|c|}{ SD } & \multicolumn{2}{|c|}{ Mean } & \multicolumn{2}{|l|}{ SD } & \\
\hline $\mathrm{WRS}^{\mathrm{a}}$ & \multicolumn{2}{|c|}{124.94} & \multicolumn{2}{|c|}{10.160} & \multicolumn{2}{|c|}{143.8} & \multicolumn{2}{|l|}{5.523} & $<0.001 *$ \\
\hline MBI-EE ${ }^{b}$ & \multicolumn{2}{|c|}{26.46} & \multicolumn{2}{|c|}{6.158} & \multicolumn{2}{|c|}{31.74} & \multicolumn{2}{|l|}{6.099} & $<0.001 *$ \\
\hline MBI-PA ${ }^{c}$ & \multicolumn{2}{|c|}{33.02} & \multicolumn{2}{|c|}{4.804} & \multicolumn{2}{|c|}{29.96} & \multicolumn{2}{|l|}{5.935} & $0.009 *$ \\
\hline \multirow[t]{2}{*}{ MBI-DP ${ }^{\mathrm{d}}$} & \multicolumn{2}{|c|}{16.32} & \multicolumn{2}{|c|}{7.229} & \multicolumn{2}{|c|}{19.45} & \multicolumn{2}{|l|}{5.321} & $0.014^{*}$ \\
\hline & Mean & SD & Median & Range & Mean & SD & Median & Range & \\
\hline Experience (years) & 12.24 & 9.895 & 4 & $1-14$ & 7.60 & 7.925 & 19 & $7-34$ & $0.009 *$ \\
\hline WCST-during ${ }^{\mathrm{e}}$ & 2.37 & 1.918 & 2 & $0-8$ & 8.40 & 3.500 & 9 & $3-15$ & $<0.001^{*}$ \\
\hline WCST-post $^{\mathrm{f}}$ & 1.00 & 1.822 & 1 & $0-12$ & 4.27 & 2.803 & 4 & $0-12$ & $<0.001^{*}$ \\
\hline
\end{tabular}

${ }^{*} P$ value $<0.05$ is considered statistically significant

${ }^{\mathrm{a}}$ Work-related stress questionnaire

${ }^{b}$ Maslach Burnout Inventory for the module of Emotional Exhaustion

${ }^{\mathrm{c}}$ Maslach Burnout Inventory for the module of Personal Achievement

${ }^{\mathrm{d}}$ Maslach Burnout Inventory for the module of Depersonalization

${ }^{\mathrm{e}}$ Wisconsin Card Sorting Test during hospital work

${ }^{\mathrm{f}}$ Wisconsin Card Sorting Test during after 2 weeks of home isolation

Table 3 Comparison of results of Mean \pm SD between married and non-married health care workers by work-related stress questionnaire (WRS), Maslach Burnout Inventory Human Services Survey (MBI-
HSS), and total errors of Wisconsin Card Sorting Test in the hospital (WCST-during) and post-hospital stay (WCST-post)

\begin{tabular}{|c|c|c|c|c|c|c|c|c|c|}
\hline & \multicolumn{3}{|c|}{ Married $(N=39)$} & & \multicolumn{3}{|c|}{ Not married $(N=42)$} & & \multirow[t]{2}{*}{$P$ value } \\
\hline & \multicolumn{2}{|c|}{ Mean } & \multicolumn{2}{|c|}{ SD } & \multicolumn{2}{|c|}{ Mean } & \multicolumn{2}{|l|}{ SD } & \\
\hline $\mathrm{WRS}^{\mathrm{a}}$ & \multicolumn{2}{|c|}{137.43} & \multicolumn{2}{|c|}{11.477} & \multicolumn{2}{|c|}{126.33} & \multicolumn{2}{|c|}{11.191} & 0.922 \\
\hline MBI-EE ${ }^{b}$ & \multicolumn{2}{|c|}{30.33} & \multicolumn{2}{|c|}{6.665} & \multicolumn{2}{|c|}{26.49} & \multicolumn{2}{|l|}{6.043} & 0.334 \\
\hline MBI-PA ${ }^{c}$ & \multicolumn{2}{|c|}{31.67} & \multicolumn{2}{|c|}{5.331} & \multicolumn{2}{|c|}{32.05} & \multicolumn{2}{|l|}{5.615} & 0.108 \\
\hline \multirow[t]{2}{*}{ MBI-DP ${ }^{\mathrm{d}}$} & \multicolumn{2}{|c|}{17.05} & \multicolumn{2}{|c|}{6.336} & \multicolumn{2}{|c|}{18.03} & \multicolumn{2}{|l|}{7.132} & 0.796 \\
\hline & Mean & SD & Median & Range & Mean & $\mathrm{SD}$ & Median & Range & \\
\hline Experience (years) & 14.46 & 10.66 & 16 & $1-34$ & 7.86 & 7.510 & 4 & $2-17$ & 0.122 \\
\hline WCST-during $^{\mathrm{e}}$ & 6.52 & 4.198 & 6 & $0-15$ & 2.54 & 2.187 & 2 & $0-11$ & $<0.001 *$ \\
\hline WCST-post ${ }^{\mathrm{f}}$ & 3.36 & 3.161 & 2 & $0-12$ & 0.97 & 1.367 & 1 & $0-6$ & $<0.001 *$ \\
\hline
\end{tabular}

${ }^{*} P$ value $<0.05$ is considered statistically significant

${ }^{a}$ Work-related stress questionnaire

${ }^{b}$ Maslach Burnout Inventory for the module of Emotional Exhaustion

${ }^{c}$ Maslach Burnout Inventory for the module of Personal Achievement

${ }^{\mathrm{d}}$ Maslach Burnout Inventory for the module of Depersonalization

${ }^{\mathrm{e}}$ Wisconsin Card Sorting Test during hospital work

${ }^{\mathrm{f}}$ Wisconsin Card Sorting Test during after 2 weeks of home isolation 
$(10 \%)$, lack of transparency of the hospital's administration (10\%) (Fig. 1).

Correlating age and experience to the perceived stress, burnout, and WCST revealed a positive significant correlation between age and each of the score of work-related stress $(r=0.578)(p<0.001)$, the score of Maslach Burnout Inventory's Module of Emotional Exhaustion, and the total errors of Wisconsin Card Sorting Test results during the stay in the hospital $(r=0.650)(p<0.001)$. However, there was no significant correlation between age and the score of Maslach Burnout Inventory's Module of Depersonalization or Module of Personal Achievement. Experience of the health care workers was negatively correlated to each of stress questionnaire $(r=-0.226)(p=0.042)$ and the total errors of Wisconsin Card Sorting Test results during hospital stay $(r=-0.227)(p=0.041)$ (Table 5).

A positive significant correlation between stress Questionnaire and the Maslach EE $(r=0.501)(p=<0.001)$ was found, in addition to expected negative correlation with Maslach PA $(r=-0.312)(p=<0.004)$. There was no significant correlation between stress Questionnaire and the Maslach DP. There was a positive significant correlation between level of stress and the total errors of Wisconsin Card Sorting Test results during the stay in the hospital $(r=0.815)(p=<0.001)$, as well as 2 weeks after stay. $(r=0.634)(p=<0.001)$ (Table 5).

There was a positive significant correlation between Maslach Burnout Inventory and the total errors of Wisconsin Card Sorting Test results during the stay in the hospital $(r=0.454)(p=<0.001)$, as well as 2 weeks after stay $(r=0.412)(p=<0.001)$ (Table 5).

Dividing HCWs according to the median $(M=135)$ of total score of stress Questionnaire on work-related stress with $51 \%(N=41)$ scoring above 135 , and $49 \%(N=40)$ below 135. Performance errors were observed in $15 \%$ $(N=6)$ of HCWs scoring above 135 , while only $2 \%(N=1)$ of Health care workers scoring below 135, which is a lower degree of work-related stress, made performance errors during their work (Fig. 2).

Nurses have shown mistakes in the administration of prescribed medications, e.g., incorrectly replacing IV steroids with oral steroids. Physicians have shown errors in the form of defective handovers or providing wrong handover reports of different patients.

\section{Discussion}

This current cohort study was carried out on 81 health care workers in Cairo University COVID-19 isolation hospitals. It was designed to assess the level of work-related stress and burnout to which the health care workers were exposed during their work in the isolation hospital. The study also assessed the sources and risk factors of work-related stress linked to the COVID-19 Pandemic. To our knowledge, this study is one of the leading studies in Egypt that objectively measured the cognitive impairment among HCWs during the 1st wave of the pandemic and whether cognitive impairment
Table 4 Comparison of results of Mean and Standard deviation between male and female health care workers in results by workrelated stress questionnaire (WRS), Maslach Burnout Inventory
Human Services Survey (MBI-HSS), and total errors of Wisconsin Card Sorting Test in the hospital (WCST-during) and post-hospital stay (WCST-post)

\begin{tabular}{|c|c|c|c|c|c|c|c|c|c|}
\hline & \multicolumn{3}{|c|}{ Male $(N=42)$} & & \multicolumn{3}{|c|}{ Female $(N=39)$} & & \multirow[t]{2}{*}{$P$ value } \\
\hline & \multicolumn{2}{|c|}{ Mean } & \multicolumn{2}{|c|}{ SD } & \multicolumn{2}{|c|}{ Mean } & \multicolumn{2}{|l|}{ SD } & \\
\hline $\mathrm{WRS}^{\mathrm{a}}$ & \multicolumn{2}{|c|}{127.29} & \multicolumn{2}{|c|}{11.55} & \multicolumn{2}{|c|}{137.26} & \multicolumn{2}{|l|}{11.65} & 0.742 \\
\hline MBI-EE ${ }^{b}$ & \multicolumn{2}{|c|}{26.67} & \multicolumn{2}{|c|}{6.513} & \multicolumn{2}{|c|}{30.44} & \multicolumn{2}{|l|}{6.244} & 0.894 \\
\hline MBI-PA ${ }^{c}$ & \multicolumn{2}{|c|}{32.57} & \multicolumn{2}{|c|}{5.518} & \multicolumn{2}{|c|}{31.08} & \multicolumn{2}{|l|}{5.313} & 0.611 \\
\hline \multirow[t]{2}{*}{ MBI-DP $^{\mathrm{d}}$} & \multicolumn{2}{|c|}{18.76} & \multicolumn{2}{|c|}{6.952} & \multicolumn{2}{|c|}{16.18} & \multicolumn{2}{|l|}{6.244} & 0.606 \\
\hline & Mean & SD & Median & Range & Mean & SD & Median & Range & \\
\hline Experience (years) & 10.71 & 9.31 & 4 & $1-27$ & 10.31 & 9.67 & 14 & $2-34$ & 0.642 \\
\hline WCST-during $^{\mathrm{e}}$ & 3.05 & 3.044 & 2 & $0-13$ & 6.28 & 4.084 & 5 & $0-15$ & $<0.001 *$ \\
\hline WCST-post ${ }^{\mathrm{f}}$ & 2.20 & 2.728 & $0-12$ & 1 & 2.92 & 2.832 & $0-12$ & 2 & $0.005^{*}$ \\
\hline
\end{tabular}

${ }^{*} P$ value $<0.05$ is considered statistically significant

${ }^{a}$ Work-related stress questionnaire

${ }^{b}$ Maslach Burnout Inventory for the module of Emotional Exhaustion

${ }^{c}$ Maslach Burnout Inventory for the module of Personal Achievement

${ }^{\mathrm{d}}$ Maslach Burnout Inventory for the module of Depersonalization

${ }^{\mathrm{e}}$ Wisconsin Card Sorting Test during hospital work

${ }^{\mathrm{f}}$ Wisconsin Card Sorting Test during after 2 weeks of home isolation 
Fig. 1 Radar plot shows different percentages of risk factors contributing to the degree of work-related stress measured

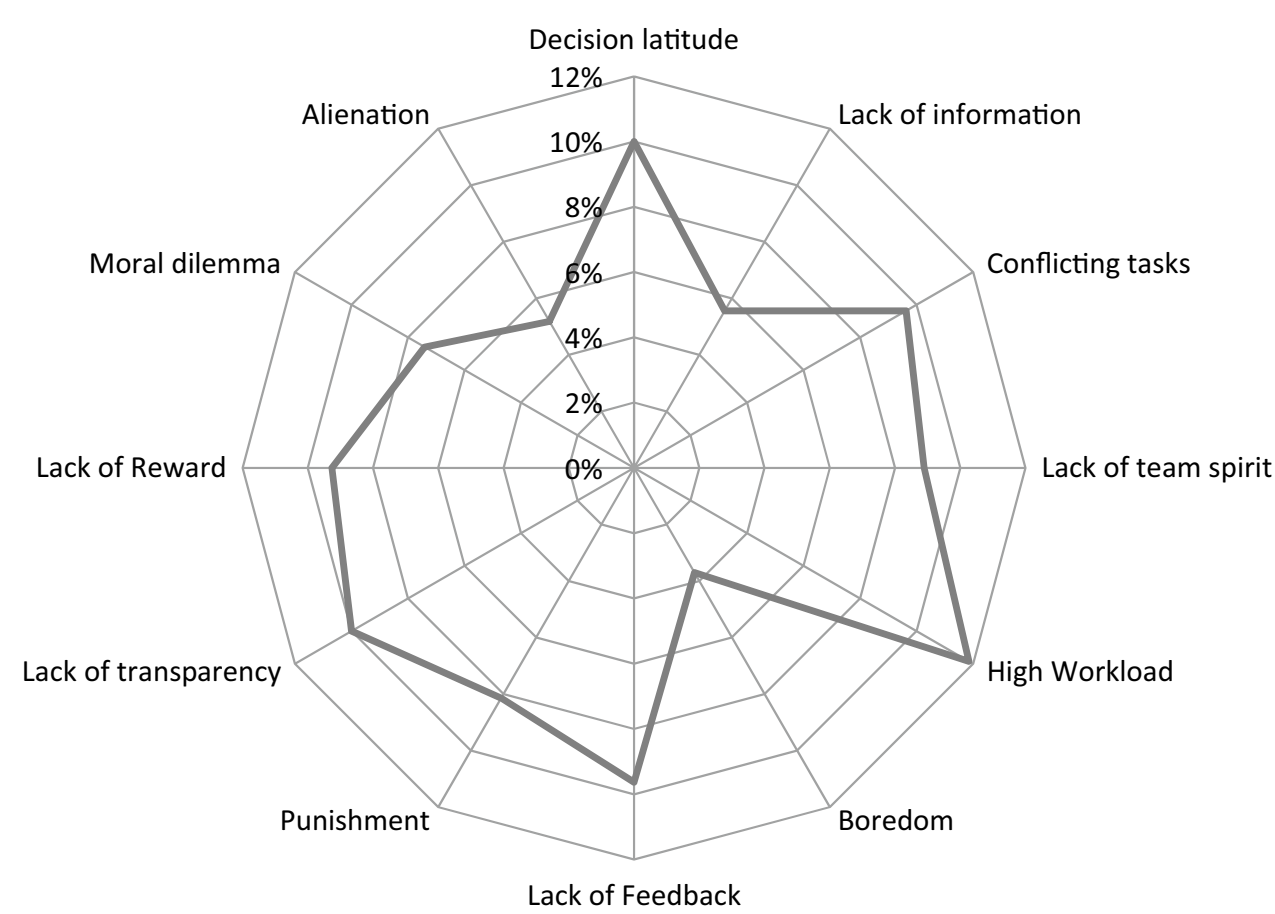

Table 5 Correlation between results of questionnaires, Wisconsin Card Sorting test, and demographic data using Spearman's test

\begin{tabular}{|c|c|c|c|c|c|c|c|c|c|}
\hline & & Age & Experience (years) & WRS & MBI-EE & MBI-DP & MBI-PA & WCST-during & WCST-post \\
\hline \multirow[t]{2}{*}{ Age } & $r$ & & 0.930 & 0.578 & 0.318 & 0.136 & -0.057 & 0.650 & 0.615 \\
\hline & $P$ & & $0.000^{*}$ & $0.000 *$ & $0.004 *$ & 0.227 & 0.616 & $0.000^{*}$ & $0.000^{*}$ \\
\hline \multirow[t]{2}{*}{ Experience (years) } & $r$ & 0.930 & & -0.226 & -0.154 & -0.207 & 0.076 & -0.227 & -0.214 \\
\hline & $P$ & $0.000^{*}$ & & $0.042 *$ & 0.170 & 0.064 & 0.501 & $0.041^{*}$ & 0.055 \\
\hline \multirow[t]{2}{*}{$\mathrm{WRS}^{\mathrm{a}}$} & $r$ & 0.578 & -0.226 & & 0.501 & 0.194 & -0.312 & 0.815 & 0.634 \\
\hline & $P$ & $0.000^{*}$ & $0.042^{*}$ & & $0.000 *$ & 0.083 & $0.005^{*}$ & $0.000^{*}$ & $0.000^{*}$ \\
\hline \multirow[t]{2}{*}{ MBI-EE ${ }^{b}$} & $r$ & 0.318 & -0.106 & 0.501 & & 0.095 & -0.135 & 0.454 & 0.412 \\
\hline & $P$ & $0.004 *$ & 0.346 & $0.000 *$ & & 0.398 & 0.230 & $0.000^{*}$ & $0.000^{*}$ \\
\hline \multirow[t]{2}{*}{ MBI-DP ${ }^{c}$} & $r$ & 0.136 & -0.143 & 0.194 & 0.095 & & -0.393 & 0.272 & 0.219 \\
\hline & $P$ & 0.227 & 0.203 & 0.083 & 0.398 & & $0.000 *$ & $0.014 *$ & $0.050 *$ \\
\hline \multirow[t]{2}{*}{ MBI-PA ${ }^{\mathrm{d}}$} & $r$ & -0.057 & 0.030 & -0.312 & -0.135 & -0.393 & & -0.280 & -0.149 \\
\hline & $P$ & 0.616 & 0.790 & $0.005^{*}$ & 0.230 & $0.000 *$ & & $0.011^{*}$ & 0.184 \\
\hline \multirow[t]{2}{*}{ WCST-during $^{\mathrm{e}}$} & $r$ & 0.650 & -0.227 & 0.815 & 0.454 & 0.272 & -0.280 & & 0.850 \\
\hline & $P$ & $0.000 *$ & $0.041^{*}$ & $0.000 *$ & $0.000 *$ & $0.014 *$ & $0.011^{*}$ & & $0.000 *$ \\
\hline \multirow[t]{2}{*}{ WCST-post $^{\mathrm{f}}$} & $r$ & 0.615 & -0.214 & 0.634 & 0.412 & 0.219 & -0.149 & 0.850 & \\
\hline & $P$ & $0.000 *$ & 0.055 & $0.000 *$ & $0.000 *$ & 0.050 & 0.184 & $0.000^{*}$ & \\
\hline
\end{tabular}

$r$ is the correlation coefficient \& it ranges from -1 to +1 ,

${ }^{*} P$ value $<0.05$ is considered statistically significant

${ }^{\mathrm{a}}$ Work-related stress questionnaire

${ }^{b}$ Maslach Burnout Inventory for the module of Emotional Exhaustion

${ }^{\mathrm{c}}$ Maslach Burnout Inventory for the module of Personal Achievement

${ }^{\mathrm{d}}$ Maslach Burnout Inventory for the module of Depersonalization

${ }^{\mathrm{e}}$ Wisconsin Card Sorting Test during hospital work

${ }^{\mathrm{f}}$ Wisconsin Card Sorting Test during after 2 weeks of home isolation 


\section{Preformance errors by health care workers during work at different degrees of work- related stress.}

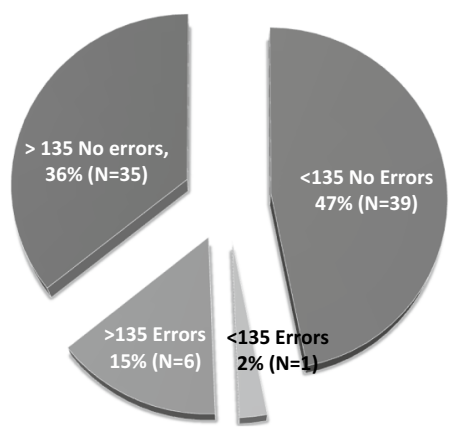

Fig. 2 The pie chart compares between HCW's score of higher workrelated stress above 135 and below 135 regarding their performance errors during work

would improve 2 weeks after leaving the hospital and had relaxed during home isolation.

The study has shown that HCWs working in the Isolation hospital were experiencing a high degree of work-related stress. The mean \pm SD of work-related stress among the study participants was $(132.08 \pm 12.573)$, which is above 98, reflecting a high level of work stress. Participants were 42 male HCWs (52\%) and 39 female HCWs (48\%). Nurses were $38 \%$ of HCWs examined, while $62 \%$ were physicians. Marital status was also taken into consideration, examining 39 married and 42 non-married HCWs.

Regarding the experienced burnout, HCWs have shown a high degree of burnout using the Maslach Burnout Inventory for Human Health Services. They have shown a mean \pm SD of $(28.48 \pm 6.622)$ for the module of Emotional Exhaustion, (31.85 \pm 5.439$)$ for Personal Achievement, and $(17.52 \pm 6.707)$ for Depersonalization. Similar results were obtained by Ismail et al. (2021), as well as Abdelhafiz et al. (2020) through their electronic survey on a sample of Egyptian frontline physicians. Within the same context, Alnazly et al. (2021) found elevated score of the fear of COVID-19 among a sample of HCWs with prevalent married females $(55 \%)$. Elevated level of fear was accompanied with depression $(40 \%)$ and anxiety $(60 \%)$.

In agreement with the current results, multiple studies emphasized that health care workers exposed to COVID-19 patients were significantly more stressed and experienced greater burnout because of the pandemic (Hall 2020; Krystal 2020). Health care workers in charge of COVID-19 patients in Wuhan and other regions in China, were also experiencing psychological burdens (Lai et al. 2020).

This was also consistent with (Kannampallil et al. 2020; Khalaf et al. 2020; Arafa et al. 2021) who used Depression,
Anxiety, Stress Scale (DASS) questionnaire to clarify that health care workers (HCWs) on the frontlines are more vulnerable to psychological distress during the COVID-19 pandemic. Nevertheless, Jahrami et al. (2021) using Perceived Stress Scale (PSS) did not observe a statistically significant difference between frontline health care workers and nonfrontline health care workers from several facilities of the Ministry of Health in Bahrain despite having a higher PSS score among the frontline workers.

It is very important to note that this study being conducted during 1st wave of the pandemic in Egypt, explained the high degree of work-related stress that can be attributed to the novelty of the disease and the lack of international guidance for the use of medications. The high workload was also due to the large number of patients admitted since patients suffering from a mild degree of COVID-19 were also admitted being put under observation (Bellanti et al. 2021).

When comparing groups of health care workers, nursing staff seemed to show a higher degree of work stress and burnout. This was in agreement with (Lai et al. 2020; Jahrami et al. 2021) who identified nurses as an occupational group who were more vulnerable to multiple stressors.

Nurses have shown a statistically significant higher degree of burnout in the form of a higher Emotional Exhaustion $(p<0.001)$ and Depersonalization $(p=0.014)$ in Maslach Burnout Inventory compared to physicians.

Nurses spend more time in the isolation hospital as compared to physicians, which can explain the higher degree of work-related stress (Caruso 2014). They spend two weeks, while physicians spend only one week. Also, nursing's pattern of shifts is based on the 12-h shift schedule. They have to be present in the high-risk zone for continuous $12 \mathrm{~h}$ only interrupted by one hour break in between, while physicians are required to check on patients twice per day while spending most of the day in their resting rooms. Although physicians are on call 24-h and they are the decision-makers during emergencies, nursing still is the occupational group that are exposed continuously for risk of infection and have to wear their PPE during the whole shift.

Khajuria et al. (2021) who used the validated Patient Health Questionnaire, also concluded that nursing staff is suffering from higher stress levels. Prasad et al. (2021) also concluded using "Coping with COVID" survey that stress is higher among nursing staff, medical assistants, and social workers.

On further analysis of the results, comparing Health care workers based on their marital status (all married health care workers in our study were having children), it was evident in our findings that the married HCWs suffered from higher degree of stress.

Arafa et al. (2021) identified the risk factor of the fear of getting infected or infecting family members was 
identified as source of stress for married health care workers, which is consistent with our findings that addressed the marital status of health care workers.

Alnazly et al. (2021) have found that the associated factors of stress experienced in the Jordanian study of HCWs during the pandemic were married, aged 40 years and older, and having more clinical experience.

In contrast, Kannampallil et al. (2020) concluded that higher burnout was experienced by unmarried health care workers based on a web survey of physicians at Washington University School of Medicine.

Regarding gender, the current study showed that there was non-significant difference in degree of work-related stress, burnout and experience between male and female HCW.

This wasn't in agreement with numerous studies that identified female HCWs as exposed to higher stress (Kannampallil et al.2020; Lai et al. 2020; Khajuria et al. 2021; Prasad et al., 2021). However, Alnazly et al. (2021) found that being male was an associated factor of stress and psychological troubles among HCWs.

When comparing health care workers in emergency shifts versus ward physicians, we did not find a significant difference in degree of work-related stress and burnout between emergency shift HCWs and ward HCW. In contrast, HCWs in emergency shifts have been identified to be predisposed to higher degree of stress (Arafa et al. 2021). Working in Intensive Care Unit (ICU) was concluded to cause elevated levels of stress (Khajuria et al. 2021).

As regards to risk factors contributing to degree of stress in the present result, it is evident through our results that risk factors contributing to development of workrelated stress are: high workload (12\%), lack of decision latitude (10\%), conflicting tasks (10\%), lack of feedback by their seniors (10\%), Lack of transparency of the hospital's administration $(10 \%)$. The second ranking risk factors were lack of team spirit (9\%), lack of Reward (9\%), presence of punishment (8\%), moral dilemmas (7\%), and lack of information (6\%). The lowest ranking risk factors were Alienation (5\%) and Boredom (4\%).

Health care workers are exposed to several risk factors inside the hospital setting that attribute to the workrelated stress; work overload, working long hours, inadequate work allocation, repetitive duties, new technology, and management issues. The psychological working environment that involves inappropriate behaviors and verbal abuse, in addition to the physical environment inside the hospital including: space, lighting, and temperature, among other causes for stress (Irfan et al. 2020). This stress might mutilate the professional efficacy, thus reducing attention, failing the decision-making skills, and decreasing concentration (Williamson 1994).
Workload as the most important risk factor is also consistent with the study done by Arafa et al. (2021) involving health care workers on the frontlines in Egypt and Saudi Arabia. Zare et al. (2021) in their study involving Iranian $\mathrm{HCW}$ s during the pandemic attained results showing that high workload was a major contributing factor to the stress level during COVID-19. Other factors examined were lack of adequate support for senior managers, their lack of preparedness to respond to emergency conditions and lack of access to PPE. Also, Irfan et al. (2020) has concluded that increased workload, low social support, organizational conflicts and acute stress reaction to death contributed to occupational stress in HCWs. Prasad et al. (2021) found that fear of exposure or transmission and work overload may be the most prominent risk factors for stress among health care workers during the pandemic. Khajuria et al. (2021) found that perceived unsatisfactory training and unavailability of PPE were important source of work stress among HCWs of 41 countries.

In the current study, HCWs with stress level higher than a score of 135 (the median) have conducted more mistakes and accidents during their practice, in the form of administering wrong medications by nurses, or handover wrong information of patients by physicians. This has also been underlined in the study of Salam et al. (2019) in Saudi hospitals, who examined the relationship between work-related stress and medication errors. This has serious implications on the performance of the whole health care system and can cause deleterious consequences on patients and HCWs themselves.

Executive functioning specifically is extremely necessary in the course of hospital work, in the form of decision making and multi-tasking. Shifting between priorities is another cognitive task required by health care workers. Therefore, Wisconsin Card Sorting Test was chosen to assess this mentioned cognitive shifting, since it's highly required in clinical settings.

Deligkaris et al. (2014) performed a systematic review exploring the relation between burnout and cognitive functioning, as assessed objectively. They have found an association between job burnout and three main cognitive functions: executive functions, attention and memory. Hendrawan et al. (2012) when investigating the efficacy of executive function tests as Wisconsin Card Sorting Test, letter fluency and Stroop test in predicting both subjective and physiological stress reactivity during acute psychosocial stress exposure, concluded that executive functioning is associated with aspects of stress regulation, specifically during acute stress exposure.

Neuropsychological assessment of stressed health care workers has revealed that they do show a degree of cognitive impairment, specifically in the domain of executive functioning. Exposure to stress activates the neural pathway supplied by catecholamines. The prolonged activation can 
have long lasting effects on the cognition (McEwen 2007). Moreover, the interaction of adrenocorticoids and adrenergic systems in specific brain regions as prefrontal cortex and the basolateral amygdala were established to explain the mechanism of actions of stress on cognition (Sandi 2013).

Our study shows that health care workers had a reduced cognitive performance while they were exposed to workrelated stress and burnout. This was confirmed by the positive significant correlation between the level of stress and the total errors of Wisconsin Card Sorting Test results during the stay in the hospital $(r=0.815)(p=<0.001)$. Another positive significant correlation between Maslach Burnout Inventory and the total errors of Wisconsin card sorting test results during the stay in the hospital $(r=0.454)$ $(p=<0.001)$ was detected to confirm our previous results.

Our findings are in accordance with many other studies examining the association of work-related stress and burnout with cognitive impairment. Burnout individuals showed impaired performance on cognitive tests used (the 2-Back and the Matching tasks) in a study of Oosterholt et al. (2012) involving 16 burnout individuals compared to 16 matched employees. Matching tasks investigate similar aspects of the executive functioning as Wisconsin Card Sorting test, namely the switching between tasks.

Eskildsen et al. (2015) concluded that individuals suffering from work-related stress do show mildly reduced performance across all the measured domains of the neuropsychological test battery. However, they did not report any statistically significant associations between selfreported perceived stress level and neuropsychological test performance.

Shields et al. (2016) used Wisconsin Card Sorting Test to assess executive functioning in stressed individuals. They had found that there are gender-specific effects on cognitive flexibility in humans establishing that men are more affected. Looking at our demographic variables as gender, our results were inconsistent with this study illustrating that female individuals show a higher cognitive impairment during exposure to stress.

Our findings do also show that the occupational group of nurses were predisposed to higher cognitive impairment as a result of exposure to work stress. Other factors as age and marital status also play a role in executive functioning. Groups of older HCWs as well as married groups do show higher total errors in WCST.

When comparing health care workers in emergency shifts versus ward physicians, we did not find significant difference in their cognitive performance. This is in agreement with Jonsdottir et al. (2013) and Maltese et al. (2016) who reported that cognitive impairment was measured in individuals with stress-related exhaustion. The most pronounced cognitive impairment examined was related to the cognitive domain of executive functioning.
In the current study, it was also concluded that the affected executive functioning of HCWs does improve after 2 weeks break from the frontline. When repeating the WCST to same HCWs after 2-week rest, it was evident that there was a significant difference in number of total errors of WCST.

\section{Limitations and strengths of the study}

Although Wisconsin Card Sorting Test is a specific assessment tool of the executive functioning domain, it cannot be concluded if the obtained cognitive underperformance is a reflection of general cognitive decline. However, full assessment necessitates the use of full neuropsychological battery which was not feasible due to the infection control measures in the isolation hospital.

In addition, pretesting of the study group before the beginning of their hospital stay would be a good baseline for their executive functioning assessment to exclude familiarity with the test. Unfortunately, the recruitment of the health care workers to the isolation hospital had been notified just few days prior to their working.

IQ is another confounding factor that can affect the WCST results among the subcategories of the study group, namely, physicians and nurses. Nevertheless, the decrease number of errors in WCST after the 2-week home isolation boosts the negative impact of the work-related stress and burnout on the cognitive functions. However, prior testing the study participants for IQ test was not feasible due to the high work load.

It is also important to point out that this sample was chosen from the isolation hospital affiliated to a faculty of medicine. However, the same study parameters need to be assessed in private sector and other local hospitals, with a larger sample size, to understand different risk factors that may contribute to work- related stress.

To our knowledge, this is the only Egyptian study that objectively, assessed the effect of work-related stress and burnout on the executive function domain using WCST, among frontline HCWs in one of isolation hospitals, during the first wave of the pandemic, with repetition of the same test after 2 weeks of home isolation. Besides this study has tried to clarify the impact of the resultant burnout and work-related stress on some professional errors that may cause deleterious consequences.

Other studies used electronic surveys to assess the degree of stress or other forms of psychological impairments, such as anxiety, depression, insomnia, and others. This carries the risk of less accurate contribution with consequent over or underestimating results. 


\section{Conclusions}

Health care workers in COVID-19 isolation hospitals experience a high degree of work-related stress. They also suffer from burnout in the form of Emotional Exhaustion, Depersonalization, and reduced Personal Achievement. Work-related stress and burnout can lead to cognitive impairment among health care workers. Female nurses and married HCWs were suffering from higher degree of work-related stress, burnout, and executive dysfunction.

Health care workers were affected by several stressors as high workload, lack of decision latitude, conflicting tasks, lack of feedback by their seniors, lack of transparency of the hospital's administration, lack of team spirit, reward and information, presence of punishment, moral dilemmas, alienation, and boredom. All of these can impact health care workers' performance leading to errors in clinical setting. Age could be one of the risk factors.

The current study points to the importance of the training workshops on the proper use of personal protective equipment (PPEs) for HCWs specially before involvement in the work in isolation hospitals. These workshops must provide adequate information about different types of PPEs, the protective role of each type, proper donning, and doffing techniques. Hand hygiene training is essential. This can decrease the fear from catching the infection. On the other hand, regular orientation sessions for HCWs should be held to clarify any updates in the treatment protocols, any change in policies or procedures in the health care facility regarding management of COVID-19 cases. Maintaining adequate number of working staff can have positive effect as it decreases the work load with consequent decrease in the amount of stress and burnout experienced by workers.

Acknowledgements The authors would like to acknowledge all the Health care workers who participated in the study for their co-operation and their valuable help.

Author contributions SAF, ORA, HSH, and MMF planned the study. SAF and MMF were accountable for the study design. HSH performed the field work of the study as a part of his MSc thesis. SAF, ORA, $\mathrm{HSH}$, and MMF provided data analysis and interpretation of the results. $\mathrm{SAF}$ and HSH conducted the statistical analysis. The manuscript draft was written by MMF. Critical review of the manuscript was provided by SAF. All authors read and approved the final manuscript.

\section{Funding None.}

Data availability Certain data will be shared on reasonable request to the corresponding author with permission of The Research Ethics Committee, Faculty of Medicine, Cairo University.

Code availability Not applicable.

\section{Declarations}

Conflict of interest None declared.

Ethics approval The Research Ethics Committee, Faculty of Medicine, Cairo University has approved the study with approval number (MS373-2020).

Consent to participate Informed consent was obtained from all the study participants

Consent to publish Not applicable.

\section{References}

Abd-Allah A, El-Hawy L (2019) Burnout and health related quality of life among resident physicians in Zagazig University Hospitals. Egypt J Occup Med 43:189-204

Abdelhafiz AS, Ali A, Ziady HH, Maaly AM, Alorabi M, Sultan EA (2020) Prevalence, associated factors, and consequences of burnout among egyptian physicians during COVID-19 pandemic. Front Public Health 8:864

Abdelkader F, Rageb EM (2010) Questionnaire on work-related stress. Anlgo-Egyptian Library. https://www.anglo-egyptian.com/ar/ book.php?id=15388. Retrieved on 14 June 2021

Alnazly E, Khraisat OM, Al-Bashaireh AM, Bryant CL (2021) Anxiety, depression, stress, fear and social support during COVID19 pandemic among Jordanian healthcare workers. PLoS ONE 16(3): 0247679

Arafa A, Mohammed Z, Mahmoud O, Elshazley M, Ewis A (2021) Depressed, anxious, and stressed: What have healthcare workers on the frontlines in Egypt and Saudi Arabia experienced during the COVID-19 pandemic? J Affect Disord 278:365-371

Bellanti F, Lo Buglio A, Capuano E, Dobrakowski M, Kasperczyk A, Kasperczyk S, Ventriglio A, Vendemiale G (2021) Factors Related to Nurses' Burnout during the First Wave of Coronavirus Disease-19 in a University Hospital in Italy. Int J Environ Res Public Health 18(10):5051

Bonde JP (2008) Psychosocial factors at work and risk of depression: a systematic review of the epidemiological evidence. Occup Environ Med 65(7):438-445

Carmassi C, Foghi C, Dell'Oste V, Cordone A, Bertelloni CA, Bui E, Dell'Osso L (2020) PTSD symptoms in healthcare workers facing the three coronavirus outbreaks: what can we expect after the COVID-19 pandemic. Psychiatry Res 292:113312

Caruso CC (2014) Negative impacts of shiftwork and long work hours. Rehabil Nurs 39(1):16-25

Deligkaris P, Panagopoulou E, Montgomery AJ, Masoura E (2014) Job burnout and cognitive functioning: a systematic review. Work Stress 28(2):107-123

Elshaer NS, Moustafa MS, Aiad MW, Ramadan MI (2018) Job stress and burnout syndrome among critical care healthcare workers. Alexandria J Med 54(3):273-277

Eskildsen A, Andersen LP, Pedersen AD, Vandborg SK, Andersen JH (2015) Work-related stress is associated with impaired neuropsychological test performance: a clinical cross-sectional study. Stress 18(2): 198-207

Grynderup MB, Mors O, Hansen ÅM, Andersen JH, Bonde JP, Kærgaard A, Kærlev L, Mikkelsen S, Rugulies R, Thomsen JF, Kolstad HA (2013) Work-unit measures of organisational justice and risk of depression-a 2-year cohort study. Occup Environ Med 70(6):380-385 
Hall H (2020) The effect of the COVID-19 pandemic on healthcare workers' mental health. JAAPA 33(7):45-48

Hasselberg K, Jonsdottir IH, Ellbin S, Skagert K (2014) Self-reported stressors among patients with exhaustion disorder: an exploratory study of patient records. BMC Psychiatry 14(1):66-76

Heaton RK, Staff PA (1993) Wisconsin card sorting test: computer version 2. Odessa Psychol Assessm Resour 4:1-4

Hendrawan D, Yamakawa K, Kimura M, Murakami H, Ohira H (2012) Executive functioning performance predicts subjective and physiological acute stress reactivity: preliminary results. Int J Psychophysiol 84(3):277-283

Irfan M, Naeem F, Afridi MI, Javed A (2020) Prevention of occupational stress in health-care workers during COVID-19 pandemic. Indian J Psychiatry 62(Suppl 3):S495

Ismail TI, Shehata SF, Mahrous RS (2021) Occupational stress and burnout among frontline Egyptian anesthesiologists during COVID-19 outbreak in Egypt. Egypt J Anaesth 37(1):91-99

Jahrami H, BaHammam AS, AlGahtani H, Ebrahim A, Faris M, AlEid K, Saif Z, Haji E, Dhahi A, Marzooq H, Hubail S (2021) The examination of sleep quality for frontline healthcare workers during the outbreak of COVID-19. Sleep Breath 25(1):503-511

Jonsdottir IH, Nordlund A, Ellbin S, Ljung T, Glise K, Währborg P, Wallin A (2013) Cognitive impairment in patients with stressrelated exhaustion. Stress 16(2):181-190

Kannampallil TG, Goss CW, Evanoff BA, Strickland JR, McAlister RP, Duncan J (2020) Exposure to COVID-19 patients increases physician trainee stress and burnout. PLoS ONE 15(8):e0237301

Khajuria A, Tomaszewski W, Liu Z, Chen JH, Mehdian R, Fleming S, Vig S, Crawford MJ (2021) Workplace factors associated with mental health of healthcare workers during the COVID-19 pandemic: an international cross-sectional study. BMC Health Serv Res 21(1):262

Khalaf OO, Khalil MA, Abdelmaksoud R (2020) Coping with depression and anxiety in Egyptian physicians during COVID-19 pandemic. Middle East Curr Psychiatry 27(1):1-7

Kohli A, Kaur M (2006) Wisconsin card sorting test: normative data and experience. Indian J Psychiatry 48(3):181

Krystal JH (2020) Responding to the hidden pandemic for healthcare workers: stress. Nat Med 26(5):639

Lai J, Ma S, Wang Y, Cai Z, Hu J, Wei N, Wu J, Du H, Chen T, Li R, Tan H (2020) Factors associated with mental health outcomes among healthcare workers exposed to coronavirus disease 2019. JAMA Netw Open 3(3):e203976

Liu Q, Luo D, Haase JE, Guo Q, Wang XQ, Liu S, Xia L, Liu Z, Yang J, Yang BX (2020) The experiences of health-care providers during the COVID-19 crisis in China: a qualitative study. Lancet Glob Health 8(6):e790-e798

Maltese F, Adda M, Bablon A, Hraeich S, Guervilly C, Lehingue S, Wiramus S, Leone M, Martin C, Vialet R, Thirion X (2016) Night shift decreases cognitive performance of ICU physicians. Intensive Care Med 42(3):393-400
Maslach C, Schaufeli WB, Leiter MP (2001) Job burnout. Annu Rev Psychol 52(1):397-422

McEwen BS (2007) Physiology and neurobiology of stress and adaptation: central role of the brain. Physiol Rev 87(3):873-904

Ng K, Poon BH, Kiat Puar TH, Shan Quah JL, Loh WJ, Wong YJ, Tan TY, Raghuram J (2020) COVID-19 and the risk to healthcare workers: a case report. Ann Intern Med 172(11):766-767

Oosterholt BG, Van der Linden D, Maes JH, Verbraak MJ, Kompier MA (2012) Burned out cognition-cognitive functioning of burnout patients before and after a period with psychological treatment. Scand J Work Environ Health 38(4):358-369

Österberg K, Karlson B, Hansen ÅM (2009) Cognitive performance in patients with burnout, in relation to diurnal salivary cortisol: original research report. Stress 12(1):70-81

Potter BA (2009) Overcoming job burnout: how to renew enthusiasm for work, 3rd edn. Ronin Publishing Inc, Oakland

Prasad K, McLoughlin C, Stillman M, Poplau S, Goelz E, Taylor S, Nankivil N, Brown R, Linzer M, Cappelucci K, Barbouche M (2021) Prevalence and correlates of stress and burnout among US healthcare workers during the COVID-19 pandemic: a national cross-sectional survey study. E Clin Med 35:100879

Salam A, Segal DM, Abu-Helalah MA, Gutierrez ML, Joosub I, Ahmed W, Bibi R, Clarke E, Qarni AA (2019) The impact of work-related stress on medication errors in Eastern Region Saudi Arabia. Int J Qual healthcare 31(1):30-35

Sandi C (2013) Stress and cognition. Wiley Interdiscip Rev Cogn Sci 4(3):245-261

Shields GS, Trainor BC, Lam JC, Yonelinas AP (2016) Acute stress impairs cognitive flexibility in men, not women. Stress 19(5):542-546

Williamson AM (1994) Managing stress in the workplace: part II-the scientific basis (knowledge base) for the guide. Int J Ind Ergon 14(1-2):171-196

Wu Z, McGoogan JM (2020) Characteristics of and important lessons from the coronavirus disease 2019 (COVID-19) outbreak in China: summary of a report of 72314 cases from the Chinese Center for Disease Control and Prevention. JAMA 323(13):1239-1242

Yu X, Wang P, Zhai X, Dai H, Yang Q (2015) The effect of work stress on job burnout among teachers: the mediating role of self-efficacy. Soc Indic Res 122(3):701-708

Zare S, Esmaeili R, Kazemi R, Naseri S, Panahi D (2021) Occupational stress assessment of healthcare workers (HCWs) facing COVID-19 patients in Kerman province hospitals in Iran. Heliyon 7(5):e07035

Publisher's Note Springer Nature remains neutral with regard to jurisdictional claims in published maps and institutional affiliations. 